\title{
Article
}

\section{NF-Y Subunits Overexpression in HNSCC}

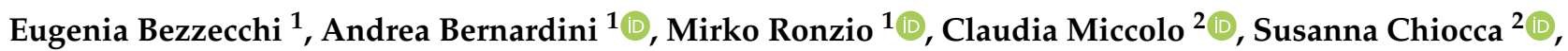 \\ Diletta Dolfini $^{1}$ (D) and Roberto Mantovani ${ }^{1, *}$ (D) \\ 1 Dipartimento di Bioscienze, Università degli Studi di Milano, Via Celoria 26, 20133 Milano, Italy; \\ eugenia.bezzecchi@unimi.it (E.B.); andrea.bernardini@unimi.it (A.B.); mirko.ronzio@unimi.it (M.R.); \\ diletta.dolfini@unimi.it (D.D.) \\ 2 Department of Experimental Oncology, IEO, European Institute of Oncology IRCCS, Via Adamello 16, \\ 20139 Milan, Italy; claudia.miccolo@ieo.it (C.M.); susanna.chiocca@ieo.it (S.C.) \\ * Correspondence: mantor@unimi.it; Tel.: +39-02-5031-5005
}

Citation: Bezzecchi, E.; Bernardini, A.; Ronzio, M.; Miccolo, C.; Chiocca, S.; Dolfini, D.; Mantovani, R. NF-Y Subunits Overexpression in HNSCC. Cancers 2021, 13, 3019. https:/ / doi.org/10.3390/cancers13123019

Academic Editor: Wollenberg Barbara

Received: 6 May 2021

Accepted: 6 June 2021

Published: 16 June 2021

Publisher's Note: MDPI stays neutral with regard to jurisdictional claims in published maps and institutional affiliations.

Copyright: (C) 2021 by the authors Licensee MDPI, Basel, Switzerland. This article is an open access article distributed under the terms and conditions of the Creative Commons Attribution (CC BY) license (https:// creativecommons.org/licenses/by/ $4.0 /)$.
Simple Summary: Cancer cells have altered gene expression profiles. This is ultimately elicited by altered structure, expression or binding of transcription factors to regulatory regions of genomes. The CCAAT-binding trimer is a pioneer transcription factor involved in the activation of "cancer" genes. We and others have shown that the regulatory NF-YA subunit is overexpressed in epithelial cancers. Here, we examined large datasets of bulk gene expression profiles, as well as single-cell data, in head and neck squamous cell carcinomas by bioinformatic methods. We partitioned tumors according to molecular subtypes, mutations and positivity for HPV. We came to the conclusion that high levels of the histone-like subunits and the "short" NF-YAs isoform are protective in HPV-positive tumors. On the other hand, high levels of the "long" NF-YAl were found in the recently identified aggressive and metastasis-prone cell population undergoing partial epithelial to mesenchymal transition, p-EMT.

Abstract: NF-Y is the CCAAT-binding trimer formed by the histone fold domain (HFD), NF-YB/NFYC and NF-YA. The CCAAT box is generally prevalent in promoters of "cancer" genes. We reported the overexpression of NF-YA in BRCA, LUAD and LUSC, and of all subunits in HCC. Altered splicing of NF-YA was found in breast and lung cancer. We analyzed RNA-seq datasets of TCGA and cell lines of head and neck squamous cell carcinomas (HNSCC). We partitioned all TCGA data into four subtypes, deconvoluted single-cell RNA-seq of tumors and derived survival curves. The CCAAT box was enriched in the promoters of overexpressed genes. The "short" NF-YAs was overexpressed in all subtypes and the "long" NF-YAl in Mesenchymal. The HFD subunits are overexpressed, except Basal (NF-YB) and Atypical (NF-YC); NF-YAl is increased in p53 mutated tumors. In HPV-positive tumors, high levels of NF-YAs, p16 and $\triangle \mathrm{Np} 63$ correlate with better prognosis. Deconvolution of single cell RNA-seq (scRNA-seq) found a correlation of NF-YAl with Cancer Associated Fibroblasts (CAFs) and p-EMT cells, a population endowed with metastatic potential. We conclude that overexpression of HFD subunits and NF-YAs is protective in HPV-positive tumors; expression of NF-YAl is largely confined to mutp53 tumors and malignant p-EMT cells.

Keywords: HNSCC; NF-Y; transcription factors; TCGA; CCAAT box; alternative splicing

\section{Introduction}

Squamous cell carcinomas of the head and neck (HNSCC) are major medical concerns worldwide [1], associated with high mortality and morbidity, as the survival outcome of these patients has remained poor over the past decades. They are a heterogenous group of tumors arising from the transformation of keratinocytes of the oral cavity and the upper respiratory tract, including the nasopharynx, paranasal sinuses, oropharynx, hypopharynx and larynx. The main risk factors are environmental (tobacco and alcohol) and human papillomavirus (HPV) infections. According to several parameters (gene expression, pathways profiling and clinical features) HNSCC is classified into four subtypes: Basal, Mesenchymal, Atypical and Classical [2-6]. 
In general, cellular transformation is a consequence of genetic mutations, entailing changes in the patterns of gene expression. This is ultimately obtained through alterations in the structure, expression levels or usage of sequence-specific transcription factors (TFs) and chromatin-modifying cofactors. Indeed, it is known that changes in the structure or expression levels of oncogenic TFs predispose, or directly cause, profound changes in gene expression, leading to tumorigenesis. Sequence-specific TFs bind to short elements within the promoters and enhancers of genes [7]. Two-decade-long efforts devoted to the discovery of genes overexpressed in different types of cancer have led to the identification of transcription factor binding sites (TFBSs) in their promoters. This was achieved by analyzing the expression profiling, first by microarrays and, more recently, by RNA-seq. In numerous reports, the CCAAT box was identified as enriched in promoters of "tumor" genes, including in a widespread analysis of $>60,000$ tumor samples [8]. This element is located in promoters at $-60 / 100$ from the transcription start sites, and a wealth of genetic studies determined that it is important or crucial for the high-level expression of genes in vitro and in vivo [9]. NF-Y is a heterotrimer formed by the histone fold domain (HFD) dimer NF-YB/NF-YC, and the sequence-specific NF-YA, providing CCAAT specificity. NF-YA is present in two major isoforms, "short" and "long", differing in 28 amino acids generated by alternative splicing of Exon 3 [10]. NF-YC is also present in multiple isoforms, resulting from alternative splicing at the $C$-terminus of the protein [11]. In both NF-YA and NF-YC, the heterogeneity involves Gln-rich trans-activation domains, whereas the subunits interaction and DNA-binding parts are identical in all isoforms. The NF-Y regulome has been studied and at least partially understood through chromatin immunoprecipitation (ChIP-Seq) and functional analysis (profiling/RNA-seq after inactivation) in different cell types. This exercise identified two common cancer-promoting pathways controlled by NF-Y: cell-cycle and metabolic genes [12-17], reviewed in [18].

NF-Y has long been considered a ubiquitous transcription factor present at similar levels in normal and transformed cells; this view has largely changed, in part because of recent studies on the mRNA expression of its subunits in cancer. Data in ovarian [19,20], breast [21] and gastric [22,23] cancers indicated overexpression of NF-YA in tumors. Examination of the levels of NF-YA in cancer specimens present in The Cancer Genome Atlas (TCGA) by Firebrowse (http: / / firebrowse.org/viewGene.html, accessed on 20 January 2020) suggested overexpression in epithelial tumors [24]. We thus started a systematic investigation of large RNA-seq datasets in cancers of epithelial origin, with analysis in the breast [24], lung [25,26] and liver [27]. Here, we focused on HNSCC. We completed the TCGA classification by including all 522 available tumors in the four molecular subtypes, we analyzed 35 HNSCC cell lines partitioned according to mutations and HPV status, we exploited single-cell RNA-seq data of primary tumors [28] to predict cell population composition of TCGA samples and we analyzed NF-Y expression. Several notable-and unexpected-features emerged.

\section{Materials and Methods}

\subsection{TCGA and CCLE RNA-Seq Dataset}

As of April 2021, RNA-seq data on 522 HNSCC primary tumors [6] and 44 non-tumor tissues were present in TCGA. We downloaded the non-normalized raw count data from http:/ / firebrowse.org/ (accessed on 20 January 2020).

To extend the currently classified TCGA tumors in the 4 molecular subtypes, we used 838 genes previously validated as signatures for the 4 individual subtypes [3]; each gene was median-centered on all 522 HNSCC samples, and Pearson correlations were calculated between the predictor centroids and the TCGA samples. A subtype prediction for each tumor was given by the centroid based on the largest correlation value.

Analysis of the RNA-seq cell lines database was performed by retrieving all available paired-end FASTQ files of the upper aerodigestive tract cell lines from the Cancer Cell Lines Encyclopedia (SRA Study SRP186687), UM-SCC-4, UM-SCC-6, UM-SCC-19, UMSCC-47 
(SRA Study SRP170971) and SCC-0472, SCC-090 (SRA Study SRP136016) from the Gene Expression Omnibus (GEO) repository. The data were processed as described above.

\subsection{Global Gene Expression and Downstream Analyses}

Cell lines RNA-seq data were analyzed with RSEM-1.3.1. to figure out expression of mRNA at genes and isoforms levels.

Differential gene expression analysis of RNA-seq data was performed using R package DESeq2 [29]. The tumor versus normal expression fold change (FC) denotes upregulation or downregulation according to the FC value. Log2FC and the corresponding false discovery rate (FDR) were reported by the R package. FDR $<0.01$ and $\mid \log _{2} \mathrm{FCl}>2$ were set as inclusion criteria for selection as a differentially expressed gene (DEG) in tumors/subtypes versus normal samples.

We used KOBAS 3.0 (http:/ / kobas.cbi.pku.edu.cn/anno_iden.php, accessed on 20 January 2020) for pathway enrichment analysis using the ENTREZ gene IDs. $p$ values of the enriched pathways were obtained by performing FDR correction. The TFBS analysis and de novo motifs were performed using Pscan software and Bonferroni corrected $p$ values, as reported in [30].

\subsection{Correlation between Mutational and HPV Status and NF-Y Levels}

We downloaded data on somatic mutations (SNP and INDEL) from the MC3 public version on https: / /tcga.xenahubs.net (accessed on 20 January 2020) and we compared NF-YA, NF-YB, NF-YC, NF-YAl and NF-YAs levels in samples with 1 or more mutations in 1 gene with the rest of samples not presenting mutations in that gene. We retrieved the HPV status in the clinical table downloaded from the firebrowse.org website. $p$ values were calculated with the Wilcoxon rank sum test.

\subsection{Western Blot Analysis}

As previously described [31], the UM-SCC-4, UM-SCC-6, UM-SCC-18, UM-SCC-19, UM-SCC-23 and UM-SCC-47 cell lines were obtained from Prof. Thomas E. Carey [32]. The UD-SCC-2 cell line was kindly provided by Prof. Henning Bier (present address: LRZ, Munich, Germany) [33]. The UM-SCC-104 cell line [34] was acquired from Merck; the UPCI:SCC-152 and UPCI:SCC-154 cell lines were from ATCC. Cells were grown in Dulbecco's modified Eagle's medium supplemented with antibiotics, $2 \mathrm{mM}$ l-glutamine, $10 \%$ fetal bovine serum and non-essential amino acids. All cell lines were authenticated by short tandem repeat profiling and tested for mycoplasma contamination every 6 months.

Total protein extracts were prepared in RIPA buffer $(150 \mathrm{mM} \mathrm{NaCl}, 1 \% \mathrm{NP}-40$, $0.5 \%$ DOC, $0.1 \%$ SDS, $50 \mathrm{mM}$ Tris- $\mathrm{HCl} \mathrm{pH} 8$ and proteases inhibitors) and equal amounts of total proteins were resolved by SDS-polyacrilamide gel electrophoresis under reducing conditions. The membrane was probed with primary antibodies and the appropriate horseradish peroxidase conjugated secondary antibodies (Sigma Aldrich, St. Louis, MO, USA). The primary antibodies used were the anti-NF-YA (G2, Santa Cruz Biotechnologies, TX, USA), anti-NF-YB (GeneSpin, Milan, Italy) and anti-Vinculin (Sigma Aldrich, St. Louis, MO, USA) as loading controls. The signal was acquired with Biorad ChemiDoc. The uncropped western blotting figure can be found in Figure S5.

\subsection{Analysis of Clinical Data}

We retrieved clinical data related to the TCGA HNSCC samples, including progressionfree interval (PFI) time records of 521 patients, from the https:/ /xenabrowser.net/ (accessed on 20 January 2020). Survival analysis of samples stratified according to NF-Y (subunit/isoforms) levels was carried out with the survminer package in the R environment, according to Kaplan-Meier analysis and log-rank test [35,36]. The two groups analyzed refer to: low < first 3 quartiles; high > last quartile. For 2-genes combination survival analysis, we independently ranked samples according to the increasing expression of genes and split them into 2 groups: the Low label corresponds to the $75 \%$ of samples 
with lower expression; High to the remaining $25 \%$. We obtained the combinations by joining the 2 rankings.

\subsection{Deconvolution Analysis of TCGA Samples and Signature Correlation}

Cell-type deconvolution on TCGA bulk RNA-seq samples was computed using the SCDC R package, using single-cell data published by Puram et al. [28] as reference datasets. For gene signature evaluation, the lists were retrieved from Puram et al. [28] and the Z-scores of the $\log _{2}$ transformed TPM (Transcripts Per Million) of genes were computed. For the CAF1/2 signatures, Z-scores were calculated on Mesenchymal samples. For the p-EMT signature samples, samples with a predicted proportion of fibroblasts lower than $30 \%$ were selected. For each signature, the median Z-score was computed and assigned to each sample. According to the median Z-score, samples were ranked and split into High (top $40 \%$ of samples) and Low (lower $40 \%$ of samples); the $20 \%$ of intermediate samples were discarded.

\subsection{Statistical Analysis}

Single comparisons between 2 groups (normal versus tumor/subtype-specific specimens and mutated versus wild-type samples) were performed with the Wilcoxon rank sum test, using the stat compare means function of the ggpubr package. In order to verify significant trends between EMT marker levels and NF-YAl/NF-YAs ratios, we divided all tumor samples into 10 groups with increasing ratio values, and then calculated the Jonckheere's Trend Test using the JT. test function provided by the SAGx package. The charts were created in the RStudio environment ( $R$ version 3.6.3) with the ggplot2 and heatmap.plus packages.

\section{Results}

\subsection{HNSCC Differentially Expressed Genes (DEGs) Have CCAAT in Their Promoters}

Nothing is known about the prevalence of CCAAT boxes in the promoters of HNSCC differentially expressed genes. We downloaded all 522 RNA-seq datasets present in TCGA [6] and compared the gene expression levels of tumors to those of the available normal samples. Using a $\mid \log _{2} \mathrm{FCl}>2$, FDR $<0.01$ threshold, 815 genes were found to be overexpressed and 1191 to be downregulated (Figure 1A), listed individually in Table S1. To gather information on regulatory DNA elements, we analyzed promoters $(-450$ to +50 from the transcriptional start site) with the Pscan software [30]. At the top of the list of overexpressed genes, we found logos of Zn Finger Transcription Factors (MZF, ZNF263, ZNF740, RREB1), with the NF-Y matrix (NFYA/NFYB) coming immediately afterward (Figure 1B, left panels). As for downregulated genes, other matrices (TBP, MEF2, FOX and NFI) were found, with robust $p$ values (Figure 1B, right panels). We then used the KOBAS software to identify Gene Ontology (GO) terms in DEGs: in upregulated genes, extracellular matrix terms (collagen formation/degradation, integrins) predominate (Figure 1C); signal transduction was enriched, but cell-cycle terms were absent, scoring a clear difference from other epithelial tumors we analyzed previously. The presence of metabolism terms among the downregulated genes is also remarkable, along with muscle, which is not immediately obvious to explain in this context.

\subsection{NF-Y Overexpression in HNSCC}

We analyzed the expression of NF-Y subunits at the gene and isoform levels (Figure 2A). We confirmed that NF-YA is indeed increased in cancer compared with normal samples $\left(p\right.$ value $\left.=10^{-14}\right)\left(\right.$ Figure 2B). Interestingly, NF-YB was increased $\left(p\right.$ value $\left.=10^{-8}\right)$ and NF-YC showed slightly elevated levels $\left(p\right.$ value $=10^{-3}$ ). Next, we analyzed the splicing isoforms of NF-YA and NF-YC. Figure 2C shows that the "short" NF-YAs were predominant in normal cells; in tumors, both NF-YA isoforms were substantially increased $\left(p\right.$ value $=10^{-9}$ ); as a consequence of this, the NF-YAl/NF-YAs ratio changed slightly in favor of NF-YAl (Figure 2D). As for NF-YC, the expression of the predominant $37 \mathrm{kD}$ NF-YC2 was not 
changed in tumors; the $50 \mathrm{kD}$ isoform, NF-YC1, increased $\left(p\right.$ value $\left.=10^{-4}\right)$ but it was still far less expressed than the $37 \mathrm{kD}$ isoform (Figure 2C).

A

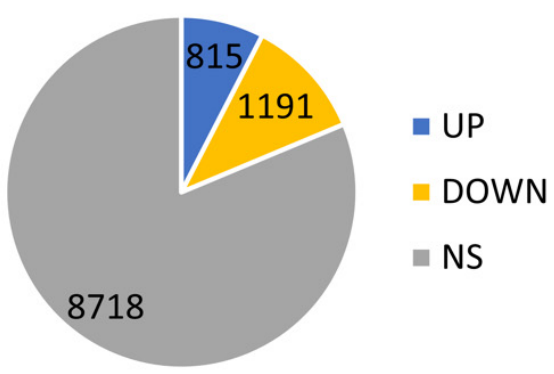

B

\begin{tabular}{|c|c|c|c|}
\hline \multicolumn{2}{|c|}{ UPREGULATED } & \multicolumn{2}{|c|}{ DOWNREGULATED } \\
\hline TF NAME & $P$ VALUE & TF NAME & P VALUE \\
\hline MZF1(var.2) & $8.38 \times 10^{-6}$ & TBP & $2.52 \times 10^{-20}$ \\
\hline ZNF263 & $8.91 \times 10^{-5}$ & MEF2A & $9.00 \times 10^{-18}$ \\
\hline ZNF740 & $1.80 \times 10^{-4}$ & Myb & $2.90 \times 10^{-13}$ \\
\hline RREB1 & $2.38 \times 10^{-4}$ & MEF2B & $4.15 \times 10^{-13}$ \\
\hline NFYA & $3.68 \times 10^{-4}$ & FOXL1 & $7.79 \times 10^{-13}$ \\
\hline$\frac{\text { NFYB }}{\text { FOS * }}$ & $\frac{4.32 \times 10^{-4}}{6.32 \times 10^{-4}}$ & GATA1::TAL1 & $1.08 \times 10^{-12}$ \\
\hline Arid3a & $9.15 \times 10^{-4}$ & MEF2D & $1.58 \times 10^{-12}$ \\
\hline TBP & $1.32 \times 10^{-3}$ & NFIA & $1.27 \times 10^{-11}$ \\
\hline TBP & $1.64 \times 10^{-3}$ & SRF & $6.95 \times 10^{-11}$ \\
\hline GATA3 & $1.85 \times 10^{-3}$ & Gata1 & $7.95 \times 10^{-11}$ \\
\hline
\end{tabular}

C

Figure 1. Gene expression analysis of HNSCC TCGA tumors. (A). Up- and downregulated genes in HNSCC versus normal tissues. NS, not significant. (B). Pscan analysis of enriched TFBS in promoters $(-450 /+50 \mathrm{bps}$ from the TSS) of up- and downregulated genes in HNSCC. (C). Reactome pathways enriched in upregulated genes (upper panel) and downregulated genes (lower panel) listed according to their $p$ value. The list was obtained using KOBAS.

Because of the concomitant increase in the levels of the three subunits, we verified whether there was a correlation of expression among them. To do so, we ranked all tumor samples for the levels of NF-YA mRNA expression, dividing them in 10 bins; we then matched the expression of NF-YB and NF-YC: as shown in Figure 2E, there was a progressive increase of these subunits from NF-YA ${ }^{\text {low }}$ to NF-YA ${ }^{\text {high }}$ expressing tumors, with a robust statistical significance. We conclude that NF-YA, NF-YB and, to a lesser extent, NF-YC levels were concomitantly increased in HNSCC with respect to normal tissues, and that the major isoforms expressed in tumors were NF-YC $37 \mathrm{kD}$ and NF-YAs, although an increase of NF-YAl was clearly recorded. 

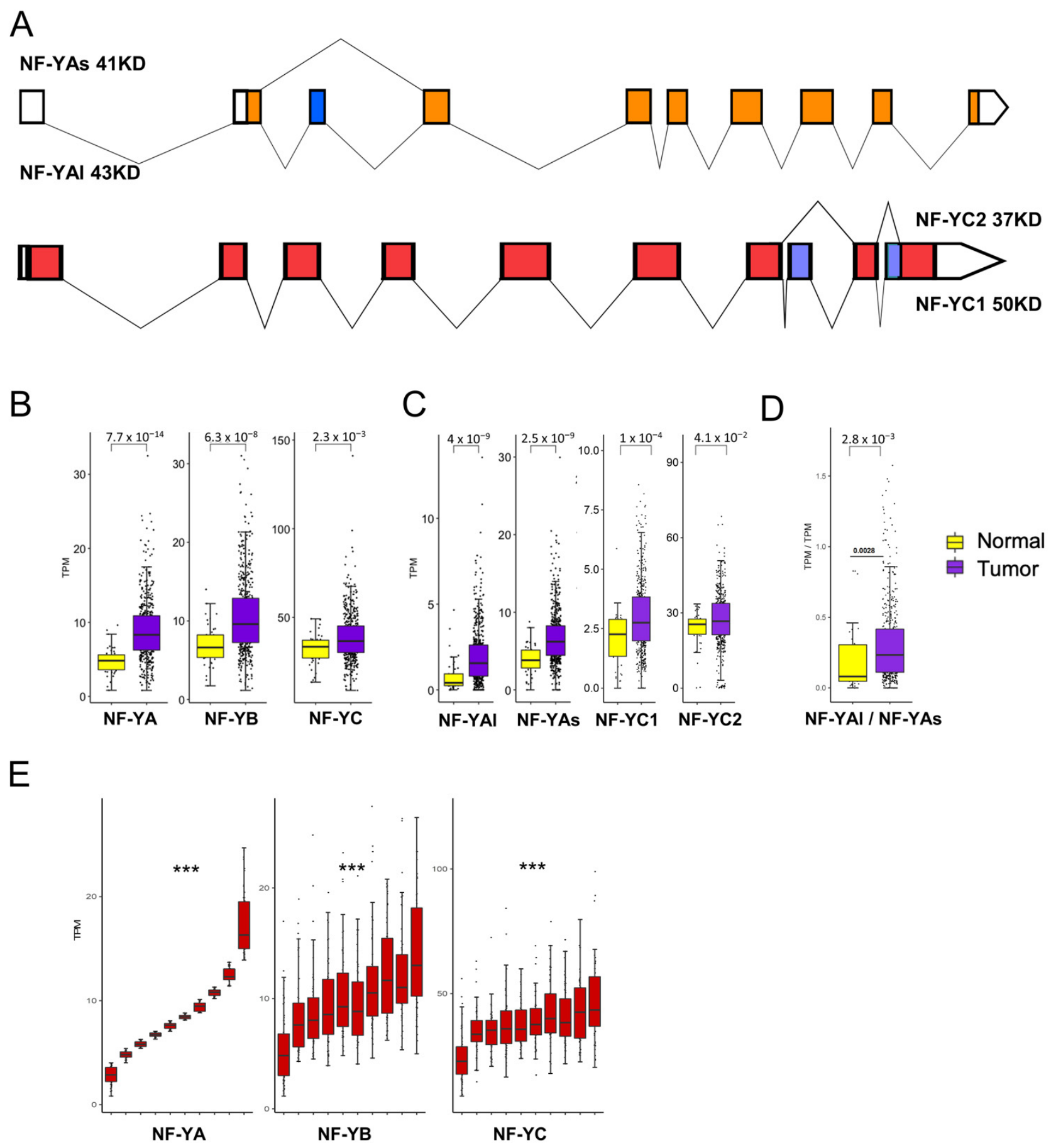

Figure 2. NF-Y subunits are overexpressed in HNSCC. (A). Scheme of NF-YA and NF-YC alternative transcripts. (B). Box plots of the expression levels of the three NF-Y subunits at gene level in the TCGA HNSCC dataset, measured in TPMs. (C). As in A, the NF-YAs, NF-YAl and NF-YC isoform levels were analyzed. NF-YC2 corresponds to the $37 \mathrm{kD}$ isoform; NF-YC1 to the $50 \mathrm{kD}$ isoform. (D). Box plots of the expression levels of the ratio of the two NF-YA isoforms. (E). Expression of NF-YB/NF-YC (right panels) according to 10 ranked bins of increasing NF-YA levels (left panels). $p$ values were calculated using the Wilcoxon rank sum test: ${ }^{* *} p$ value $<0.0001$.

\subsection{Expression of NF-Y Isoforms in HNSCC Cell Lines}

To correlate the mRNA and protein levels of the isoforms, particularly NF-YAs and NF-YAl, we analyzed their relative levels in RNA-seq data from 35 HNSCC cell lines: the vast majority (26) had a large excess of NF-YAs: only Hs840T, YD8 and SNU46 had higher 
amounts of NF-YAl (Figure 3A). NF-YB mRNAs were relatively well balanced, with the exception of higher levels in YD8. As for NF-YC, the pattern is relatively uniform, with most lines lacking, or having very low expression of the $50 \mathrm{kD}$ (NF-YC1) isoform.

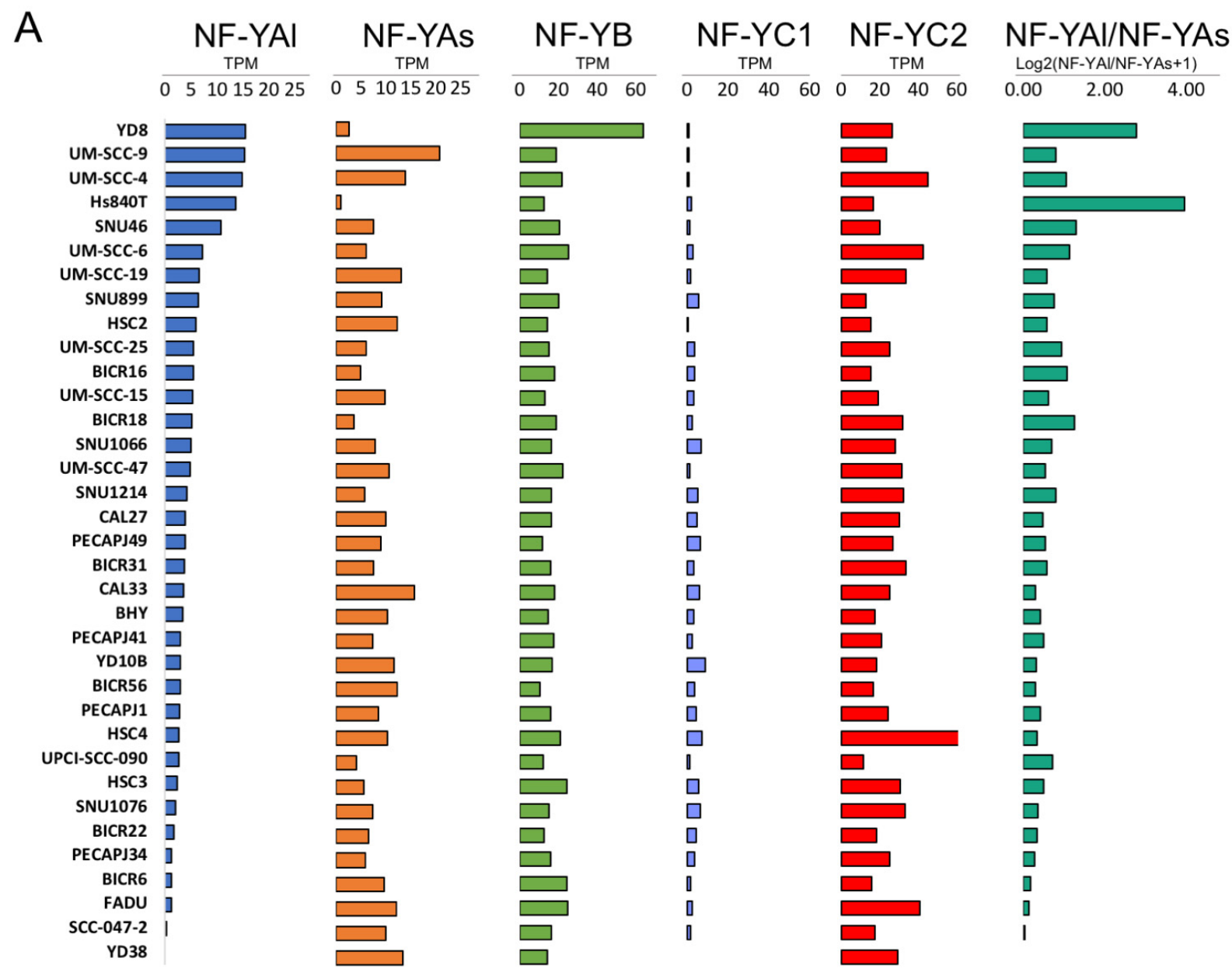

B

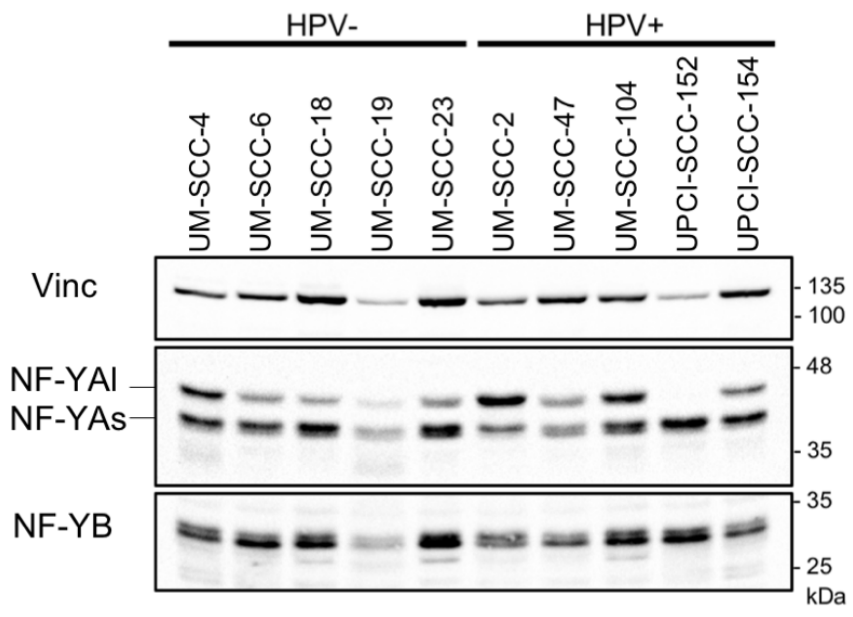

Figure 3. Expression levels of NF-Y mRNA and protein isoforms in HNSCC cell lines. (A). Expression levels of NF-Y subunits (expressed in TPMs) and NF-YA isoforms ratio in 35 cell lines of HNSCC. (B). Western blot analysis of NF-YA and NF-YB in the indicated HNSCC cell lines. 
We performed western blot analysis of the NF-Y subunits in several HNSCC cell lines, both positive and negative for HPV. As shown in Figure 3B, cells showed variable levels of the two NF-YA isoforms, with a prevalence of the short isoform. For UM-SCC-4, UM-SCC-6, UM-SCC-19 and UM-SCC-47, a comparison with the available RNA-seq data (Figure 3A) indicated good concordance: NF-YAs was predominant in UM-SCC-19 and UM-SCC-47, and balanced levels were observed in UM-SCC-4 and UM-SCC-6. Overall, there was no obvious skewing according to the HPV status of the lines. As for NF-YB, we noticed some variation in the protein levels among the lines, somewhat superior to the relatively invariant mRNA levels. Altogether, these data indicate that there is a good agreement between the mRNA and isoforms levels in HSNCC cell lines.

\subsection{Analysis of NF-Y Subunits Levels According to Somatic Mutations in HNSCC}

TCGA identified somatic mutations in 506 tumors [6]. Here, we considered only the 12 most frequent mutations present in $>80$ samples (Figure S1), assessing the NF-Y subunits expression in their relative cohorts (Figure 4). Mutations of CDKN2A (p16) and NOTCH1 were associated with a statistically relevant decrease in NF-YAs, NF-YB and NF-YC; the same was seen in TP53 mutants, but in this case with a substantial increase in NF-YAl. Other changes, less statistically significant, were an increase in NF-YB in CSMD3, an increase in NF-YAs in TTN and CSMD3, and a decrease in NF-YC in PCLO mutants. TCGA has compiled a list of cancer driver genes [37] and the Ciccarelli group has further refined this by pointing out potential false positive drivers [38], based on numerous validation criteria (http:/ / ncg.kcl.ac.uk/false_positives.php, accessed on 10 May 2020). We analyzed the above data and noted that altered expression of NF-Y subunits was most obvious in samples with mutations of bona fide drivers (TP53, CDKN2A, NOTCH1, FAT1) but less so in samples with potential "false positive" mutations. We concluded that selected cancerdriving mutations are associated with altered expression, both positive and negative, of NF-Y subunits.

\subsection{Classification of All TCGA HNSCCs in Four Subtypes}

Overexpression of NF-Y subunits could be limited to one or more of the HNSCC subtypes. TCGA has so far reported the partitioning of 279 of the 522 tumors for which RNA-seq data are available [6]. As we have previously done in our analysis of breast, lung and liver cancers, before proceeding with further analysis, we classified all TCGA tumors; to do so, we used the gene signature described by Walter et al. [3]. Table S2 contains our classification of all TCGA tumors. Figure S2A shows the results, depicted as Venn diagrams, of the old and our extended classification: the Atypical and Mesenchymal classes maintain the same proportions, but modest skewing is present in Basal and in Classical, the least frequent subtype. A heatmap of our classification was constructed based on a predictor centroid of 838 genes derived from the Walter classification [3]: this is shown in Figure S2B, confirming a visible clustering of the four major subtypes. In particular, Figure S2C shows the expression levels of typical markers of each subtype [3,6]: Atypical (RPA2, E2F2, FGFR3), Classic (SOX2, NFE2L2, KEAP1, PIC3CA, AKR1C1) and Mesenchymal (PDFGRA, PDGFRB, TWIST1) were partitioned as expected. Of the three basal markers, only TGF-A showed the expected pattern, while the expression of TP63 was higher in classic tumors (Figure S2C). Overall, our extended classification of all TCGA HNSCC tumors was robust and it represented the basis for further analysis. 

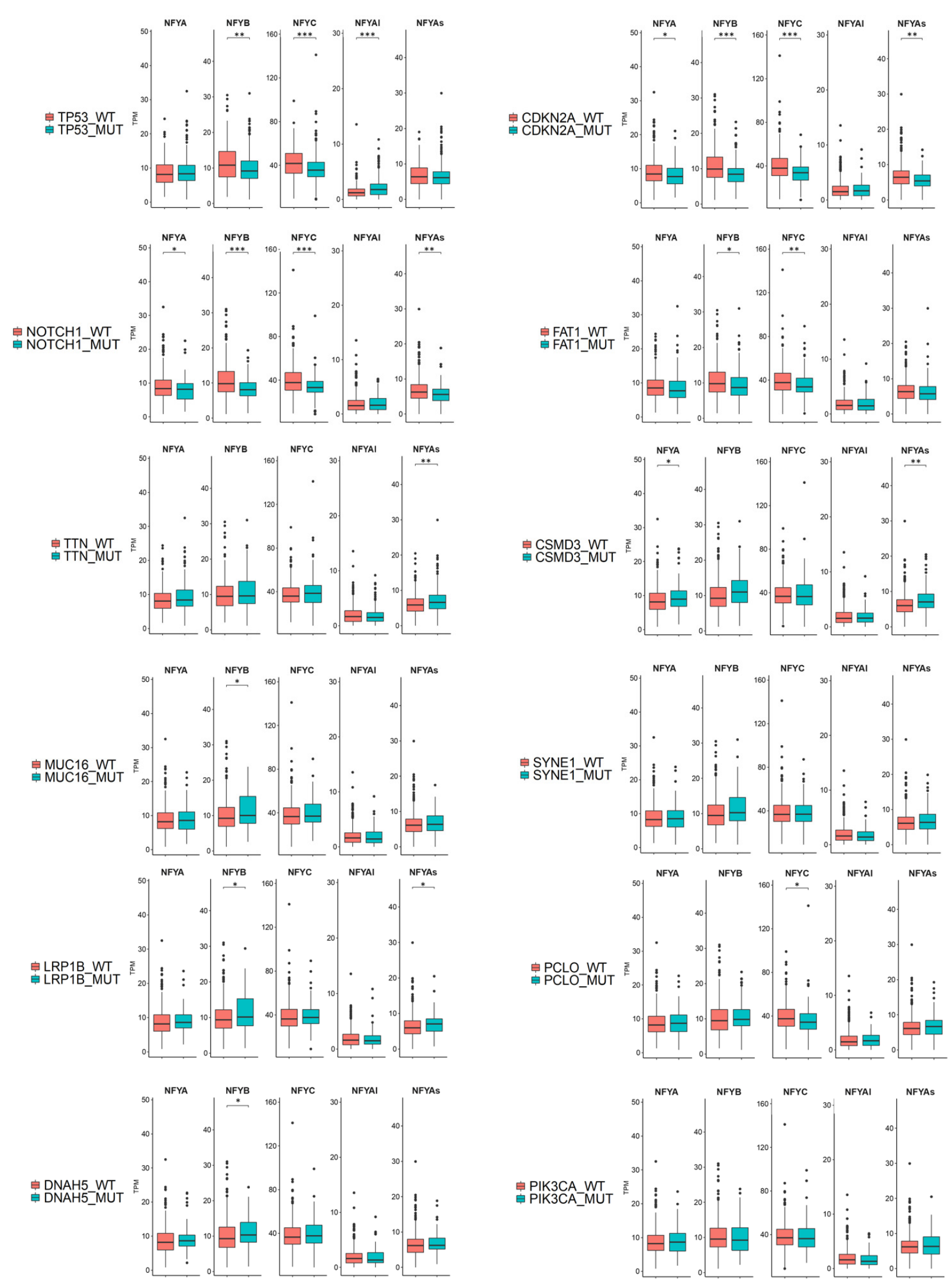

Figure 4. Expression levels of NF-Y isoforms according to common mutations in HNSCC. Relative levels of expression of the NF-Y subunits and isoforms (TPMs) in HNSCC tumors presenting the 12 most common mutations, as indicated in the different Panels. $p$ values were calculated using a Wilcoxon signed-rank test. ${ }^{*} p$ value $<0.05 ;{ }^{* *} p$ value $<0.01 ;{ }^{* * *} p$ value $<0.001$. 


\subsection{Expression of NF-Y Isoforms in HNSCC Subtypes}

With the complete HNSCC RNA-seq dataset on hand, we performed individual analyses of the RNA-seq of the four subtypes. Venn diagrams of the overlaps are shown in Figure S3A (left panel), and lists of the differentially expressed genes are presented in Table S2. The common set of 257 genes upregulated in all subtypes lacks NF-Y sites (Figure S3A right panel) and retains the features described in the global analysis, notably extracellular matrix terms (Figure S3A bottom panel). As for subtype-specific transcription factor binding sites, distinct matrices were enriched, with $p$ values being borderline significant, except for Mesenchymal subtype (Figure S3B). We then analyzed the Gene Ontology terms enriched in individual subtypes (Figure S3C): the signatures of Classical and Atypical subtypes have low statistical significance; Basal and Mesenchymal show the expected terms: extracellular matrix and collagen in the latter, cornified envelope and keratinization in the former (Figure S3C). Overall, while the CCAAT box was enriched in promoters of genes overexpressed in HNSCC at large (Figure 1), it was not in specific subtypes.

We then investigated the expression levels of the three subunits. Figure $5 \mathrm{~A}$ shows a global increase in NF-YA in all subtypes, with somewhat variable degrees ( $p$ values $\leq 10^{-8 / 16}$ ). NFYB increased at a comparable level in all except Basal tumors; NF-YC was unchanged, except in Atypical tumors, where it is significantly ( $p$ value $=10^{-7}$ ) increased (Figure 5A). The data on NF-YA and NF-YC isoforms are shown in Figure 5B: NF-YAs increased in all, particularly in Atypical tumors ( $p$ values $=10^{-5 / 10}$ ); interestingly, the most robust increase was scored for NF-YAl in Mesenchymal tumors $\left(p\right.$ value $\left.=10^{-14}\right)$. As a consequence, the NF-YAl/NF-YAs expression ratio is changed substantially in this subtype ( $p$ value $=10^{-7}$ ) (Figure 5C). As for NF-YC, the increased levels were mostly due to the less expressed NF-YC1 $50 \mathrm{kD}$ isoform in Atypical tumors (Figure 5B).

In previous studies on breast and lung tumors, the NF-YAl/NF-YAs ratios were clinically more useful than the levels of the single isoforms. Specifically, partitioning of BRCA cell lines in clusters according to their NF-YAl/NF-YAs ratios, and determining the respective differentially expressed genes, allowed us to derive useful predictor centroids, which were subsequently validated in tumors [24]. This was not possible with HNSCC cell lines, due to the paucity of those with high levels of NF-YAl (Figure 3). We then decided to take a different approach and directly partitioned all samples in 10 bins, according to the ranking of their NF-YAl/NF-YAs ratios. Figure 5D shows that tumors with high ratios (NF-YAl ${ }^{\text {high }}$ ) were mostly $(65-69 \%)$ of the Mesenchymal subtype, while Atypical tumors were only $4-5 \%$. On the contrary, tumors with low ratios (NF-YAs ${ }^{\text {high }}$ ) were mostly (48-49\%) Atypical, with very few Mesenchymal tumors. Basal and Classical tumors had intermediate NF-YAl/NF-YAs ratios.

In summary, overexpression of NF-YA and NF-YB was generally widespread, with some specificities: NF-YAl/NF-YAs ratios were high in Mesenchymal tumors, due to increased levels of NF-YAl, and low in Atypical tumors, with no increase in NF-YB in Basal and an increase in NF-YC isoforms in Atypical tumors.

\subsection{Analysis of HPV-Positive Tumors}

A considerable number of HNSCC, mostly oropharyngeal cancers, are associated with infection with human papilloma virus (HPV) [39-42], as reviewed in [43-46]. Firstly, we verified that the $98 \mathrm{HPV}$-positive tumors present in our new classification were mostly classified as Atypical tumors (65\%, Figure 6A), as previously shown by TCGA [6]. We then determined the expression of NF-Y subunits in this cohort. The box plots of global subunits levels in HPV-negative and HPV-positive tumors versus normal samples showed a generalized increase (Figure 6B, left panels). In the central panels, we scored the NF-YA isoforms: NF-YAl was lower, NF-YAs was higher and, as a consequence, the NF-YAl/NFYAs ratio dropped in HPV-positive tumors (Figure 6B, right panel). In parallel, we identified differentially expressed genes in this cohort, with the same criteria used above. Figure 6C shows that 813 genes were overexpressed and 1214 were downregulated. TFBS analysis by Pscan detected the NF-Y matrix at the top in upregulated genes, with E2F and the sites 
of KLF5, Znf740, EGR1/4 and Sp1 (Figure 6D, left panels). In downregulated genes, the promoters had other matrices, such as TATA, MEF2 and NFI (Figure 6D, right panels). As for GO pathways, together with the extracellular matrix terms found in the general analysis, we found cell-cycle genes, which was expected based on the concomitant presence of the NF-Y and E2F matrices in the promoters (Figure S4). Therefore, we detected a distinct gene expression profile in HPV-positive tumors, with CCAAT, E2F and motifs of cell-cycle genes; NF-YAs and NF-YB/NF-YC subunits were clearly overexpressed. Note that these features are different from those scored in Atypical tumors at large.

A

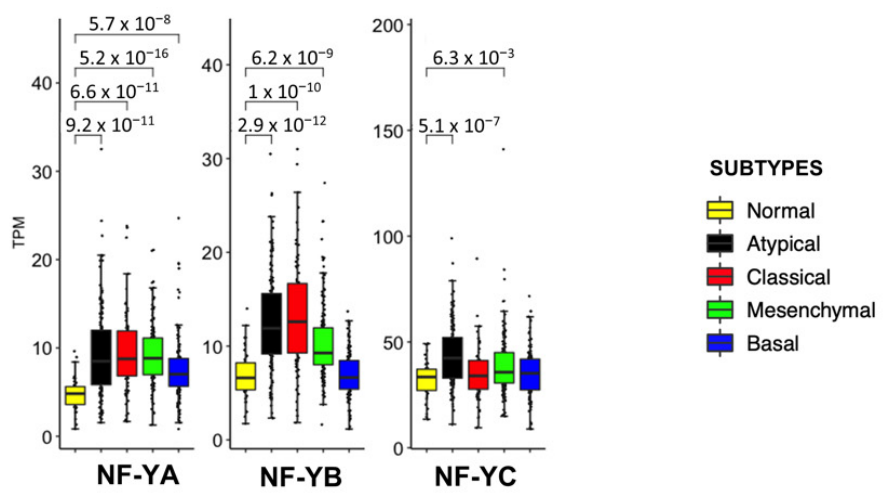

B
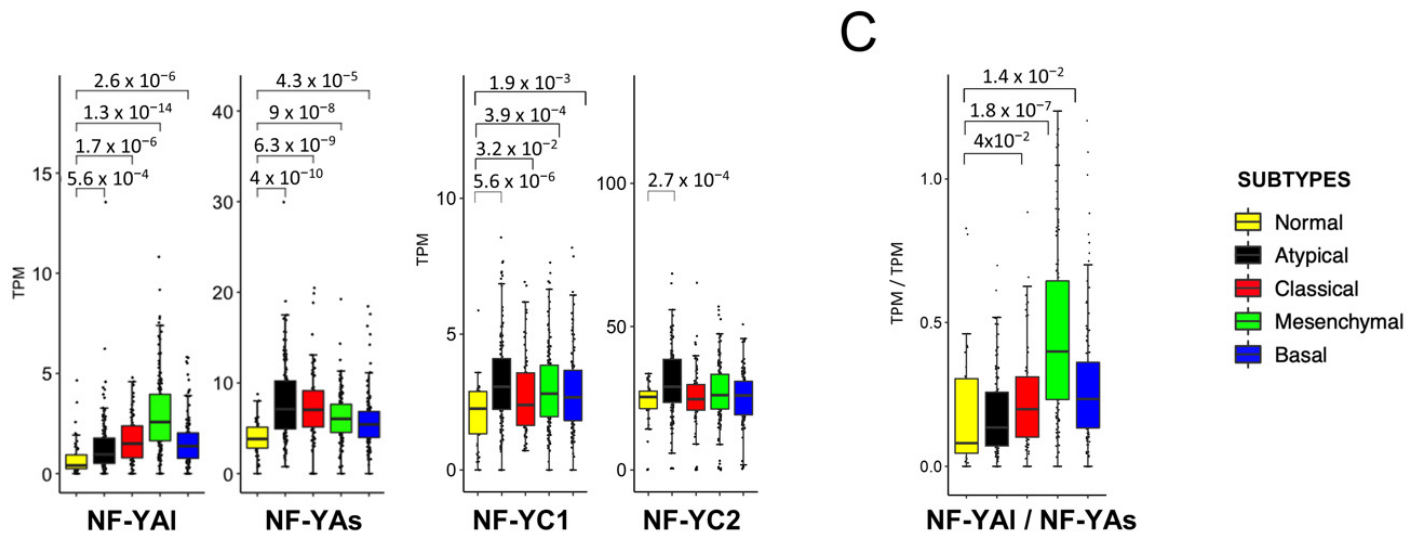

D

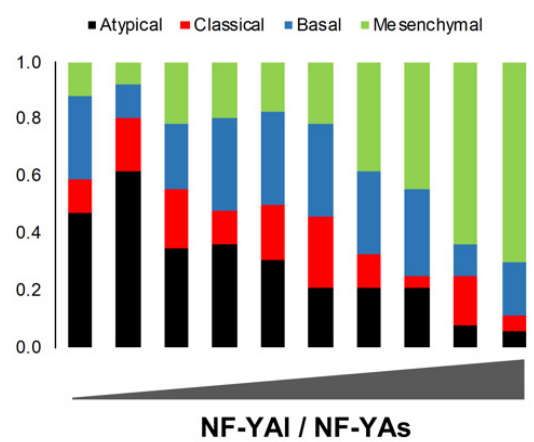

Figure 5. NF-Y subunit expression in HNSCC subtypes. (A). Box plots of expression levels of the three NF-Y subunits at gene level in the four subtypes and normal samples of the TCGA HNSCC dataset, measured in TPMs. (B). As in A, with the NF-YAs, NF-YAl, NF-YC1 and NF-YC2 subunits. (C). Box plots of the expression levels of the ratio of the two NF-YA isoforms. $p$ values were calculated using the Wilcoxon rank sum test. (D). Partitioning of HNSCC subtypes according to increasing NF-YAl/NF-YAs ratios, divided into 10 bins. 
A

HPV positive TCGA tumors

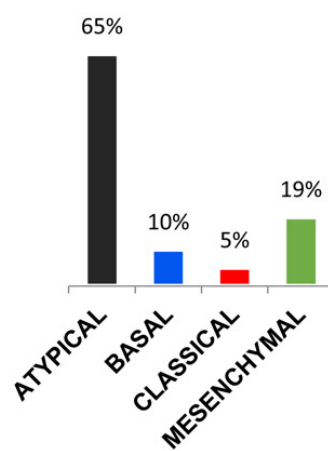

C

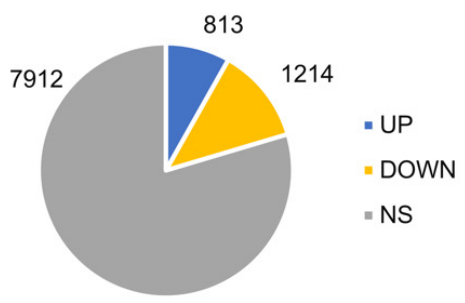

E

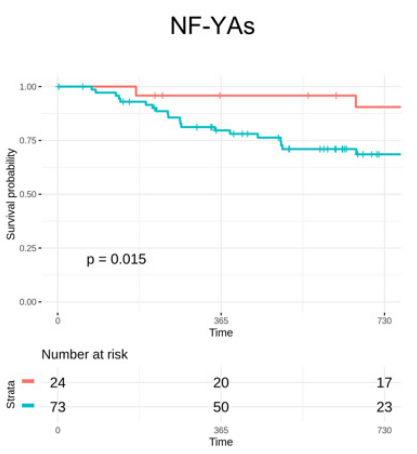

B
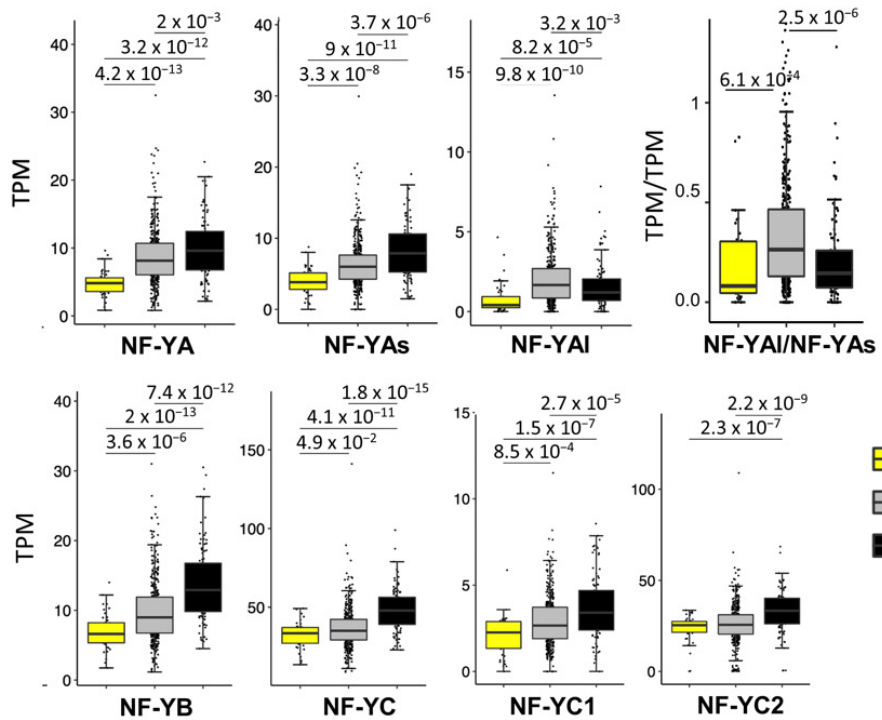

官 Normal

官 Negative

Positive

D

\begin{tabular}{|c|c|c|c|}
\hline \multicolumn{2}{|c|}{ UPREGULATED } & \multicolumn{2}{c|}{ DOWNREGULATED } \\
\hline TFNAME & PVALUE & TFNAME & $P$ VALUE \\
\hline NFYA & $1.38 \times 10^{-7}$ & TBP & $1.62 \times 10^{-26}$ \\
\hline E2F6 & $1.57 \times 10^{-7}$ & MEF2A & $1.80 \times 10^{-18}$ \\
\hline KLF5 & $2.75 \times 10^{-7}$ & NFIA & $8.21 \times 10^{-15}$ \\
\hline NFYB & $5.24 \times 10^{-7}$ & PKNOX2 & $3.62 \times 10^{-14}$ \\
\hline ZNF740 & $6.29 \times 10^{-7}$ & MEF2A & $4.17 \times 10^{-14}$ \\
\hline EGR1 & $2.30 \times 10^{-6}$ & GATA1::TAL1 & $4.28 \times 10^{-14}$ \\
\hline E2F4 & $3.30 \times 10^{-6}$ & PKNOX1 & $2.14 \times 10^{-13}$ \\
\hline E2F1 & $3.87 \times 10^{-6}$ & MEF2B & $2.45 \times 10^{-13}$ \\
\hline EGR4 & $5.58 \times 10^{-6}$ & TGIF1 & $4.18 \times 10^{-13}$ \\
\hline SP1 & $1.30 \times 10^{-5}$ & MEF2A & $1.62 \times 10^{-12}$ \\
\hline E2F3 & $1.49 \times 10^{-5}$ & TGIF2 & $3.06 \times 10^{-12}$ \\
\hline
\end{tabular}

F

NF-YAs/CDKN2A
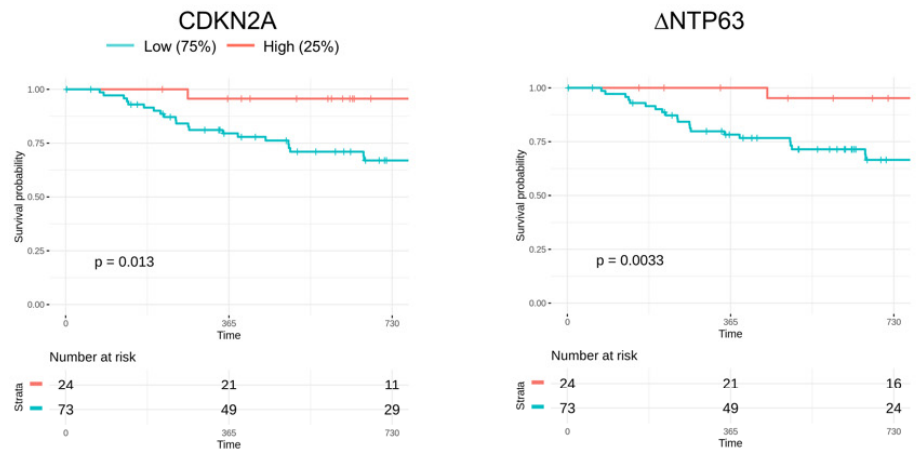

NF-YAs/ANTP63
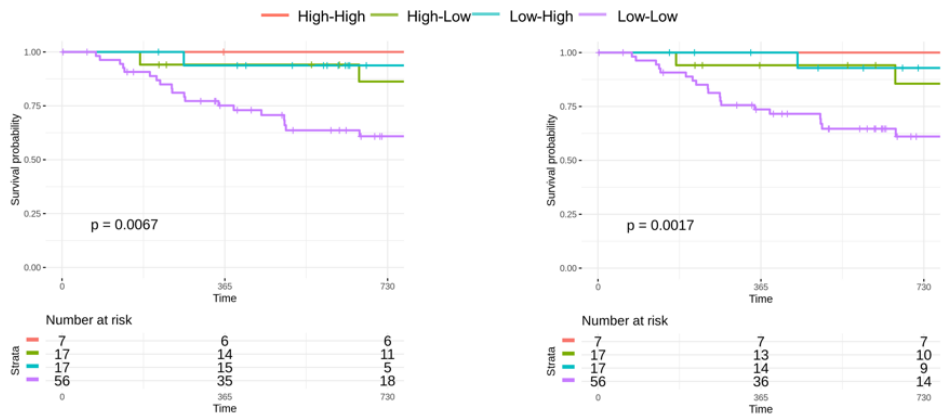

Figure 6. Analysis of NF-Y subunits expression in HPV-positive HNSCC. (A). Partitioning of all TCGA HNSCC subtypes 
according to HPV positivity. (B). Box plots of the expression levels of NF-Y subunits at gene and isoform levels in the TCGA HPV-positive and HPV-negative HNSCC, measured in TPMs. $p$ values were calculated using the Wilcoxon rank sum test. (C). Up- and downregulated genes in HPV-positive versus normal tissues. NS, not significant. (D). Pscan analysis of enriched TFBS in promoters $(-450 /+50 \mathrm{bps}$ from the TSS) of up- and downregulated genes in HPV-positive HNSCC tumors. (E). Progression-free interval curves of the survival probability of HNSCC tumors with stratification according to the expression of NF-Y subunits. (F). Progression-free interval curves of the survival probability of HNSCC tumors with stratification according to the combination of expression of NF-YAs and CDKN2A or $\triangle$ NTP63.

We then considered the clinical data and measured the PFI of HPV-positive tumors. We stratified the data according to the levels of NF-Y subunits (top 25\% versus lowest 75\%): NF-YA total and NF-YAl were not significant, but NF-YAs correlated with a better prognosis (Figure 6E, left panel). As positive controls, we evaluated CDKN2A, whose high levels are known to be protective [6], as well as $\Delta \mathrm{Np} 63$, which we recently demonstrated to be increased in HPV-positive by the E6 viral protein [47]; both genes are indeed very significantly protective (Figure $6 \mathrm{E}$, middle and right panels). Finally, pairwise combination of curves of NF-YAs with CDKN2A or $\triangle$ Np63 further confirmed this point (Figure 6F). We concluded that overexpression of NF-YAs in HPV-positive tumors correlated with a CCAAT-driven, proliferative signature associated with better patient outcomes.

\subsection{Deconvolution of Single-Cell RNA-Seq Analysis}

All TCGA data were from bulk tumors, which are intrinsically heterogeneous in terms of cellular populations. Hence, it was not possible to determine precisely which population expressed the NF-Y isoforms. Puram et al. reported on an analysis of single-cell RNA-seq from HNSCC [28], providing a breakdown of the TCGA classification and introducing important concepts: (i) the prevalence of fibroblasts in Mesenchymal tumors; (ii) the presence of p-EMT cells, specifically in Basal and Mesenchymal tumors; and (iii) the identification of gene signatures, including those for p-EMT and cancer-associated fibroblasts (CAFs).

Fibroblasts are known to mainly express NF-YAl [10]; thus, it was important to assess whether the abundance of NF-YAl found in Mesenchymal tumors (Figure 5B) could be ascribed exclusively to this population of cells. To solve the issue, we deconvoluted the single-cell RNA-seq data and partitioned the subtypes according to the cellular components (cancer cells, fibroblasts, myocytes, macrophages etch). The results are shown in Figure 7A: cancer cells, which are prevalent, were equally distributed in all subtypes, whereas fibroblasts were specifically abundant in the Mesenchymal subtype, as expected from Puram's analysis. We then analyzed all TCGA samples, after elimination of those particularly abundant in fibroblasts ( $>30 \%$ of cellularity) according to the deconvolution: Figure 7B shows that indeed most, but not all, of the evicted samples belong to the Mesenchymal subtype. We partitioned the remaining samples according to their p-EMT signature (High and Low), as described by Puram et al. [28]: the box plots of Figure 7C (left panels) show that a high NF-YAl and NF-YAl/NF-YAs ratio, but not NF-YAs, correlated with the signature. In their analysis of TCGA data, Puram et al. focused on the Basal and Mesenchymal subtypes; we thus repeated the analysis focusing on these two subtypes, excluding Classical and Atypical tumors, and indeed found a correlation very similar to the global analysis (Figure 7C, right panels). Therefore, NF-YAl ${ }^{\text {high }}$ and a high NF-YAl/NF-YAs ratio were associated with cancer-promoting cells with a partial mesenchymal phenotype.

Finally, we analyzed NF-YA isoforms levels in cancer-associated fibroblasts of the Mesenchymal subtype, as defined by Puram' CAF1 and CAF2 specific gene signatures. We observed a correlation with high NF-YAl levels, as well as the NF-YAl/NF-YAs ratio, for both signatures (Figure 7D). In conclusion, we showed that high NF-YAl, but not NF-YAs, was associated both with p-EMT cells and CAFs within tumors in vivo. 
A
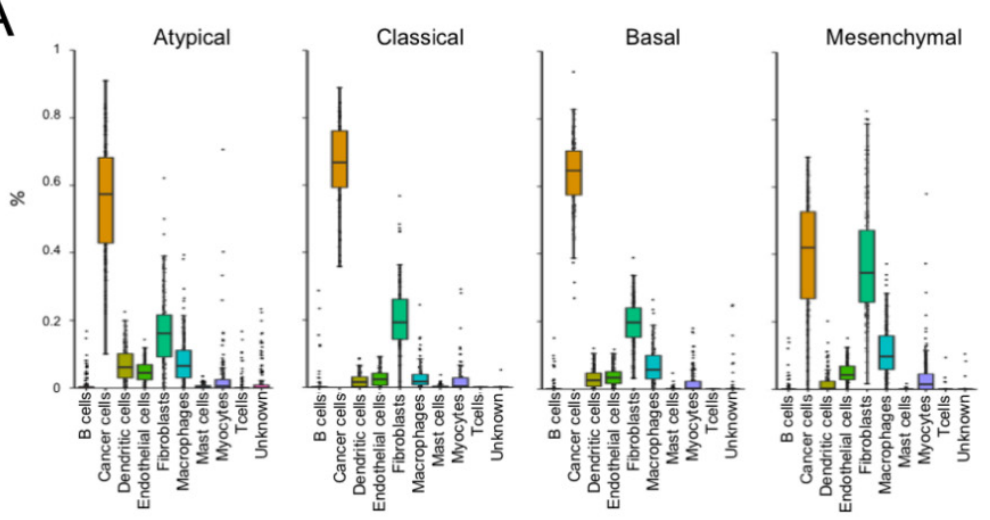

B

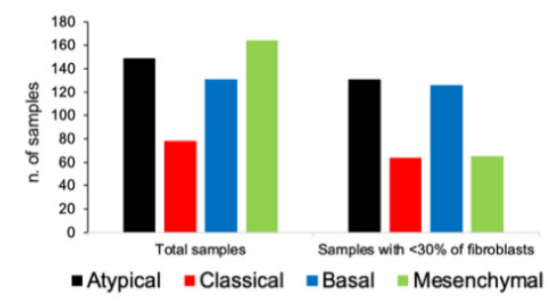

C

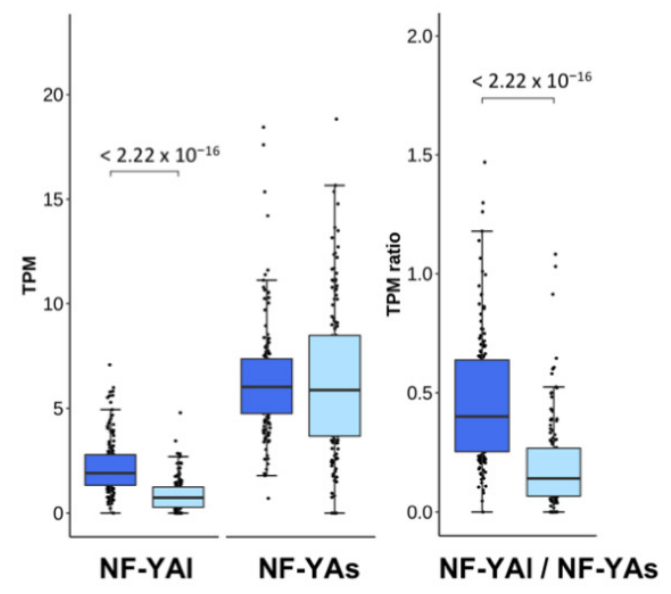

D
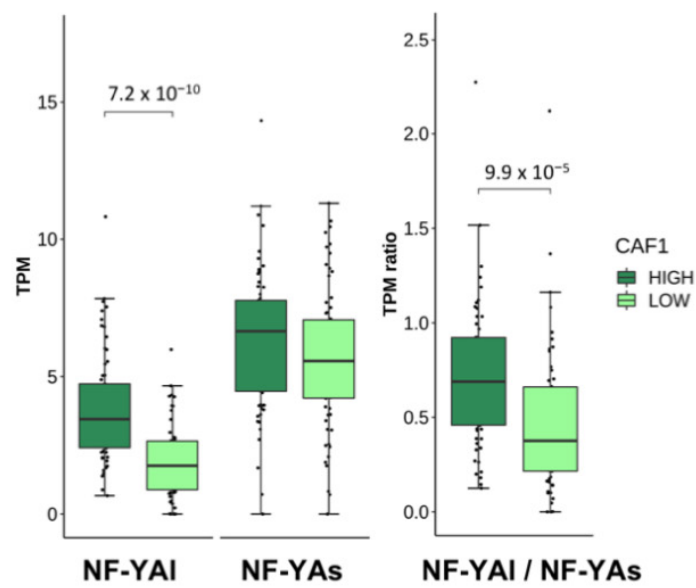
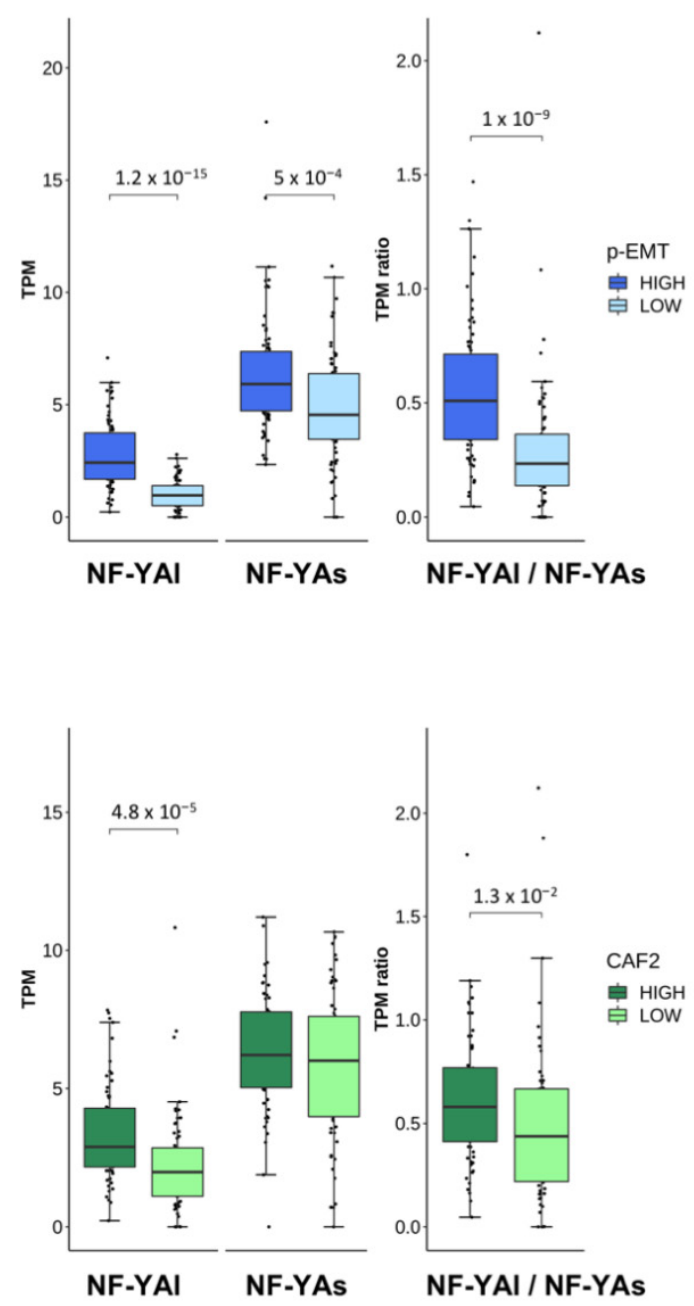

Figure 7. Correlation of NF-YA isoforms according to deconvolution of the scRNA-seq analysis. (A). Proportion of the individual cell populations, as predicted based on scRNA-seq deconvolution of tumors, within the TCGA HNSCC subtypes. (B). Subtype distribution of all TCGA tumor samples before (left panel) and after (right panel) elimination of samples with a predicted fibroblasts population of $>30 \%$. (C). Left panels: box plots of NF-YA isoforms and NF-YAl/NF-YAs ratio, according to the p-EMT signature: total TCGA samples are divided into high and low. Right panels: As in C, except that the analysis was limited to Basal and Mesenchymal tumors of the TCGA classification. (D). As in C (left panels), except that CAF1 (left panels) and CAF2 (right panels) signatures were used in the total TCGA samples. $p$ values were calculated using the Wilcoxon rank sum test. 


\section{Discussion}

The increased expression of NF-YA in epithelial tumors is becoming obvious, as other Authors have shown [19-23], and as we have progressively been reporting in our systematic TCGA-based analysis [24-27]. The results shown here further confirm the abundance of NF-Y sites (CCAAT) in the promoters of genes overexpressed in cancer, the increased expression of the "short" NF-YA in epithelial tumors and of "long" NF-YA in tumors expressing mesenchymal markers. In addition, we found (i) global overexpression of the NF-YB/NF-YC subunits, with clinical implications, and (ii) the association of selected somatic mutations and HPV infection with altered expression of the subunits. Most importantly, deconvolution of single-cell RNA-seq led us, for the first time, to associate NF-YAl expression with the p-EMT subpopulation.

CCAAT boxes have been routinely found in promoters of genes overexpressed in cancer, first in microarray profiling experiments [8] and subsequently in RNA-seq data. CCAAT genes are associated with pro-proliferative GO terms, either globally (breast) or in subpopulations, such as in the lung and liver [24-27]. We found them here in HPVpositive tumors, in agreement with NF-Y being essential for cell-cycle and metabolic genes associated with tumorigenesis [18]: CCAAT was found with E2Fs sites, in line with their role and overexpression in cancers. In HNSCC at large, instead, we found extracellular matrix/collagen and general mesenchymal terms in upregulated genes. E2Fs motifs were not enriched. This might reflect the abundance of fibroblasts, primarily, but not exclusively, in Mesenchymal tumors. As expected, CCAAT was not found in the promoters of genes downregulated in HNSCC: despite their frequency in promoters, and their recent inclusion in the DNA elements that govern transcriptional start site selection [48], this confirmed that promoter configurations of "cancer" genes—with CCAAT—are structurally different from downregulated genes, which are often CCAAT-less.

A notable difference with respect to our analysis in breast and lung cancers is that NF-YB and, to a lesser extent, NF-YC are overexpressed in tumors. The number of NF-YB mRNA molecules appears to approach that of NF-YC, the most expressed of the subunits. This has a precedent in liver cancers [27] and in one study on NF-YC in gliomas [49]. Overexpression of NF-YB could impact cancer cells, as this subunit was shown to have a pro-survival effect, in partnership with E2F1 [50]. Regulation of the localization of HFD subunits might also be relevant. No specific data are available on HNSCC lines, but NF-YB is intrinsically nuclear in other epithelial cells, while NF-YC is also cytoplasmatic, being transferred to the nucleus through NF-YB dimerization [51,52]. Thus, higher NF-YB levels could increase heterodimer formation in the nucleus, which is, in turn, met by higher levels of NF-YA to form functional CCAAT-binding trimers. Note that a direct relationship between increased levels of NF-YA mRNA and protein levels has been documented in gastric cancer [23] and we have shown that this was also observed in HNSCC cell lines. Furthermore, expression of the NF-YC protein is affected by the levels of the oncogenic lncRNA SNHG3 in SCC-9 cells, via decoying of the RNA-binding protein ELAV1L [53].

NF-Y subunits are rarely mutated in tumor samples. We evaluated the association of NF-Y levels with mutations frequently detected in HNSCC and noted the link with p53. NF-YAs and HFD subunits are comparatively less expressed in p53, NOTCH1 and CDKN2A (p16) mutated tumors. Furthermore, we reported an increase in NF-YAl. As for p53, 60-65\% of HNSCC are mutated [4-6,54-56], whereas squamous cell carcinomas of the skin and lung reach $85-90 \%$ [57]. Infection by the HPV virus is a powerful driving force in the pathogenesis of HNSCC, particularly those of the oropharynx, with approximately 70\% being HPV16-positive [38-41,45]. A growing body of genetic and clinical evidence indicates that HPV-positive tumors are different from HPV-negative tumors. They carry wtp53, while the vast majority ( $>80 \%$ ) of HPV-negative tumors are mutated in p53 [6]. HPV produces viral proteins (E6, E7) which inactivate the function of wtp53 by several mechanisms [43-45]. In essence, p53 in HNSCC is either mutated or inactivated by viral proteins. Both mechanisms were shown to impact on the NF-Y regulome: p53 mutations, specifically those with gain-of-function, directly activate several NF-Y targets [58-63]. As 
for viral proteins, NF-Y activates the E6 promoter of HPV18 in HeLa cells, via a proximal promoter CCAAT box [64]. It remains to be seen whether an increased level of NF-Y induces HPV16 E6/E7 expression. In HPV-positive tumors, expression of all subunits was substantially increased, and positive feedback could increase the oncogenic potential of E6/E7 proteins. Inactivation of E6 reactivates wtp53 protein, causing either direct apoptosis or sensitizing cells to anti-cancer drugs [65-67]. Another player is the related p63, specifically the $\Delta \mathrm{Np} 63$ isoform, which is predominant in squamous cell carcinomas; $\mathrm{p} 63$ is a master gene of stratified epithelia [68], whose role in HPV-positive tumors is not entirely clear. We recently showed that E6 activates the expression of $\triangle \mathrm{Np} 63$ in HPV-positive cells [46], and found a correlation here with NF-YB/NF-YC/ / NF-YAs. The promoter of $\triangle \mathrm{Np63}$ contains two CCAAT elements and is regulated by NF-Y [69]: we are thus tempted to speculate that E6 and the increased NF-Y levels lead to overexpression of $\Delta$ Np63. In turn, increased expression of p63 would maintain epithelial identity, preventing epithelial to mesenchymal transition: this would be in line with the clinical data of Figure 6, showing the worst prognosis in patients whose $\triangle \mathrm{Np} 63$ and NF-YAs levels are low. At the opposite end, high levels of mutp53, as found in most HPV-negative tumors, could subvert the expression of the epithelial markers normally controlled by $\Delta \mathrm{Np} 63$, with loss of epithelial identity. A further issue is the co-regulation of the three subunits. In HPV18-positive HeLa cells, removal of NF-YA by RNAi leads to increased HFD levels, and, vice versa, NF-YB inactivation entails upregulation of NF-YA [64]. In HPV-positive tumors, all three subunits are upregulated: thus, the feedback loop is apparently interrupted. The role of viral proteins on subunit levels, mutp53 and $\Delta \mathrm{Np} 63$ awaits further biological confirmation.

The two major NF-YA splicing isoforms differ in the Gln-rich trans-activation domain (TAD), with NF-YAl harboring 28/29 extra amino acids coded by Exon 3: both isoforms retain subunit interaction and DNA-binding domains, leading to the indistinguishable CCAAT-binding of the isoforms. They have different activation potential $[10,70]$. Short NF-YA is the most abundant, both in normal tissue and in HNSCC. This isoform is also predominant in all epithelial cancers we have examined so far: we are starting to believe that this is linked to the epithelial identity of the cells. A subset of Claudin ${ }^{\text {low }}$ breast tumors in which NF-YAl predominates corresponds to the most aggressive, EMT-prone subset. Indeed, it was recently proposed that the Claudin ${ }^{\text {low }}$ group represents a fifth subtype in BRCA [71]. We found a similar situation in the HNSCC Mesenchymal subtype, with a correlation between EMT markers and NF-YAl ${ }^{\text {high }}$ and high NF-YAl/NF-YAs ratios.

TCGA provides data from bulk tumors. The seminal study of Puram et al. called for a simplification of the current classification, indicating that Basal and Mesenchymal subtypes could be classified into a single subtype, with Mesenchymal being a subclass with an abundant component of stromal cells [28]. Most importantly, the study showed evidence of the existence of p-EMT cells, located at the leading edge of tumors. They also described gene signatures for CAFs. By deconvoluting single-cell RNA-seq data with the use of the p-EMT and CAF signatures, we established a correlation between NF-YAl, but not NF-YAs, and the typical markers of CAFs, which are important components of the tumor microenvironment, including in squamous cell carcinomas [72]. We also provide the first indication that p-EMT, a metastasis-prone population, correlates with NF-YAl, and their NF-YAl/NF-YAs ratios are different from other subtypes (Atypical, Classic). This confirms and extends Puram's study, which called for the modification of the current classification of HNSCC based on four molecular subtypes. Our results also have potential diagnostic value: on one hand, we have reinforced the necessity of deriving tools to carefully assess the pEMT signature in primary tumors, which is apparently more revealing than other features based on the current molecular classification; on the other, quantification of the single NFYA isoforms by RT-PCR might be valuable in the assessment, respectively, of HPV-positive (NF-YAs ${ }^{\text {high }}$ / protective) and HPV-negative (NF-YAl ${ }^{\text {high }} /$ deleterious) tumors. 


\section{Conclusions}

We have pointed to the role of NF-YAs/HFD in the prognosis of wtp53 HPV-positive tumors and of NF-YAl in p-EMT (Basal/Mesenchymal) cells. Mechanistically, as most EMT genes are CCAAT-less, NF-YAl's role might be indirect, via activation of EMT-inducing $\mathrm{TF}(\mathrm{s})$. We should extend our findings to the $\mathrm{p}-\mathrm{EMT} / \mathrm{NF}-\mathrm{YAl}$ connection with other tumors through single-cell RNA-seq data (BRCA, in primis), as well as identifying connections with EMT TF(s). Impairment of NF-Y activity could be desirable in HPV-negative tumors, and attempts in this direction have recently started [73,74].

Supplementary Materials: The following are available online at https://www.mdpi.com/article/10 .3390 / cancers13123019/s1, Figure S1. Tumor mutations in HNSCC TCGA data. Available genetic data on 316 TCGA HNSCC were portioned according to the individual mutations, as shown in the table. Figure S2. A. Venn diagram of the TCGA HNSCC dataset with the four subtypes previously categorized [3]. Each circle shows the number with the previous partial partitioning and the new classification presented here. B. Heatmap with clustering of HNSCC samples in four subtypes, according to the 838 gene centroids, kindly provided by V. Walter [3]. C. Box plots of relative mRNA levels of 14 marker genes typical of the four subtypes, as indicated by the color: black (Atypical), green (Mesenchymal), red (Classical), blue (Basal). Figure S3. Analysis of HNSCC subtype-specific gene expression. A. Venn diagrams of upregulated genes in the different subtypes of HNSCC. Right panel: Pscan analysis of enriched TFBS in promoters of the common cohort of upregulated genes. Lower panel: Reactome analysis of the upregulated genes. B. Pscan analysis of enriched TFBS in promoters of the subtype-specific upregulated genes, as indicated by the Venn diagrams. C. Reactome pathways enriched in upregulated genes in the four subtypes of HNSCC, listed according to their $p$ value. The list was obtained using KOBAS. Figure S4. Pathways enriched in HPV tumors. Reactome pathways enriched in HPV-positive upregulated genes (upper panel) and downregulated genes (lower panel) listed according to their $p$ value. The list was obtained using KOBAS. Figure S5: The uncropped western blotting figure. Table S1. Differentially expressed genes. Table S2. Classification of TCGA samples.

Author Contributions: D.D. and R.M. designed the experiments. E.B., A.B., M.R. and C.M. performed and analyzed the experiments. S.C., R.M. and D.D. wrote the manuscript. All authors have read and agreed to the published version of the manuscript.

Funding: This work was supported by AIRC Grant 19050 to R.M. and Ministero della Salute GR2013-02355625 to D.D.

Institutional Review Board Statement: Not applicable.

Informed Consent Statement: Not applicable.

Data Availability Statement: The data presented in this study are available on request from the corresponding author.

Acknowledgments: We thank P. Gandellini and N. Gnesutta for comments and critical reading of the manuscript. We are indebted to B.E. Bernstein for communicating the details of the single-cell analysis published by Puram et al. [28].

Conflicts of Interest: The authors declare no conflict of interest.

\section{References}

1. Siegel, R.; Naishadham, D.; Jemal, A. Cancer Statistics 2012. CA Cancer J. Clin. 2012, 62, 10-29. [CrossRef]

2. Chung, C.H.; Parker, J.S.; Karaca, G.; Wu, J.; Funkhouser, W.K.; Moore, D.; Butterfoss, D.; Xiang, D.; Zanation, A.; Yin, X.; et al. Molecular classification of head and neck squamous cell carcinomas using patterns of gene expression. Cancer Cell 2004, 5, 489-500. [CrossRef]

3. Walter, V.; Yin, X.; Wilkerson, M.D.; Cabanski, C.R.; Zhao, N.; Du, Y.; Ang, M.K.; Hayward, M.C.; Salazar, A.H.; Hoadley, K.A.; et al. Molecular subtypes in head and neck cancer exhibit distinct patterns of chromosomal gain and loss of canonical cancer genes. PLoS ONE 2013, 8, e56823. [CrossRef]

4. Stransky, N.; Egloff, A.M.; Tward, A.D.; Kostic, A.D.; Cibulskis, K.; Sivachenko, A.; Kryukov, G.V.; Lawrence, M.S.; Sougnez, C.; McKenna, A.; et al. The Mutational Landscape of Head and Neck Squamous Cell Carcinoma. Science 2011, 333, 1157-1160. [CrossRef] [PubMed] 
5. $\quad$ Pickering, C.; Zhang, J.; Yoo, S.Y.; Bengtsson, L.; Moorthy, S.; Neskey, D.M.; Zhao, M.; Alves, M.V.O.; Chang, K.; Drummond, J.; et al. Integrative Genomic Characterization of Oral Squamous Cell Carcinoma Identifies Frequent Somatic Drivers. Cancer Discov. 2013, 3, 770-781. [CrossRef]

6. The Cancer Genome Atlas Network. Comprehensive genomic characterization of head and neck squamous cell carcinomas. Nature 2015, 517, 576-582.

7. Levine, M.; Cattoglio, C.; Tjian, R. Looping Back to Leap Forward: Transcription Enters a New Era. Cell 2014, 157, 13-25. [CrossRef] [PubMed]

8. Goodarzi, H.; Elemento, O.; Tavazoie, S. Revealing Global Regulatory Perturbations across Human Cancers. Mol. Cell 2009, 36, 900-911. [CrossRef]

9. Dolfini, D.; Mantovani, R.; Zambelli, F.; Pavesi, G. A perspective of promoter architecture from the CCAAT box. Cell Cycle 2009, 8, 4127-4137. [CrossRef]

10. Li, X.; van Huijsduijnen, R.H.; Mantovani, R.; Benoist, C.; Mathis, D. Intron-exon organization of the NF-Y genes. Tissue-specific splicing modifies an activation domain. J. Biol. Chem. 1992, 267, 8984-8990. [CrossRef]

11. Ceribelli, M.; Benatti, P.; Imbriano, C.; Mantovani, R. NF-YC Complexity Is Generated by Dual Promoters and Alternative Splicing. J. Biol. Chem. 2009, 284, 34189-34200. [CrossRef] [PubMed]

12. Linhart, C.; Elkon, R.; Shiloh, Y.; Shamir, R. Deciphering Transcriptional Regulatory Elements That Encode Specific Cell-Cycle Phasing by Comparative Genomics Analysis. Cell Cycle 2005, 4, 1788-1797. [CrossRef]

13. Halperin, Y.; Linhart, C.; Ulitsky, I.; Shamir, R. Allegro: Analyzing expression and sequence in concert to discover regulatory programs. Nucleic Acids Res. 2009, 37, 1566-1579. [CrossRef]

14. Fleming, J.D.; Pavesi, G.; Benatti, P.; Imbriano, C.; Mantovani, R.; Struhl, K. NF-Y coassociates with FOS at promoters, enhancers, repetitive elements, and inactive chromatin regions, and is stereo-positioned with growth-controlling transcription factors. Genome Res. 2013, 23, 1195-1209. [CrossRef] [PubMed]

15. Xie, D.; Boyle, A.; Wu, L.; Zhai, J.; Kawli, T.; Snyder, M. Dynamic trans-Acting Factor Colocalization in Human Cells. Cell 2013, 155, 713-724. [CrossRef]

16. Dolfini, D.; Zambelli, F.; Pedrazzoli, M.; Mantovani, R.; Pavesi, G. A high definition look at the NF-Y regulome reveals genomewide associations with selected transcription factors. Nucleic Acids Res. 2016, 44, 4684-4702. [CrossRef] [PubMed]

17. Benatti, P.; Chiaramonte, M.L.; Lorenzo, M.; Hartley, J.A.; Hochhauser, D.; Gnesutta, N.; Mantovani, R.; Imbriano, C.; Dolfini, D. NF-Y activates genes of metabolic pathways altered in cancer cells. Oncotarget 2016, 7, 1633-1650. [CrossRef]

18. Gurtner, A.; Manni, I.; Piaggio, G. NF-Y in cancer: Impact on cell transformation of a gene essential for proliferation. Biochim. Biophys. Acta Bioenerg. 2017, 1860, 604-616. [CrossRef] [PubMed]

19. Mamat, S.; Ikeda, J.; Tian, T.; Wang, Y.; Luo, W.; Aozasa, K.; Morii, E. Transcriptional Regulation of Aldehyde Dehydrogenase 1 A1 Gene by Alternative Spliced Forms of Nuclear Factor Y in Tumorigenic Population of Endometrial Adenocarcinoma. Genes Cancer 2011, 2, 979-984. [CrossRef]

20. Cicchillitti, L.; Corrado, G.; Carosi, M.; Dabrowska, M.E.; Loria, R.; Falcioni, R.; Cutillo, G.; Piaggio, G.; Vizza, E. Prognostic role of NF-YA splicing isoforms and Lamin A status in low grade endometrial cancer. Oncotarget 2016, 8, 7935-7945. [CrossRef]

21. Yang, C.; Zhao, X.; Cui, N.; Liang, Y. Cadherins Associate with Distinct Stem Cell-Related Transcription Factors to Coordinate the Maintenance of Stemness in Triple-Negative Breast Cancer. Stem Cells Int. 2017, 2017, 1-13. [CrossRef]

22. Cao, B.; Zhao, Y.; Zhang, Z.; Li, H.; Xing, J.; Guo, S.; Qiu, X.; Zhang, S.; Min, L.; Zhu, S. Gene regulatory network construction identified NFYA as a diffuse subtype-specific prognostic factor in gastric cancer. Int. J. Oncol. 2018, 53, 1857-1868. [CrossRef] [PubMed]

23. Bie, L.-Y.; Li, D.; Mu, Y.; Wang, S.; Chen, B.-B.; Lyu, H.-F.; Han, L.-L.; Nie, C.-Y.; Yang, C.-C.; Wang, L.; et al. Analysis of cyclin E co-expression genes reveals nuclear transcription factor $\mathrm{Y}$ subunit alpha is an oncogene in gastric cancer. Chronic Dis. Transl. Med. 2019, 5, 44-52. [CrossRef]

24. Dolfini, D.; Andrioletti, V.; Mantovani, R. Overexpression and alternative splicing of NF-YA in breast cancer. Sci. Rep. 2019, 9, 1-12. [CrossRef] [PubMed]

25. Bezzecchi, E.; Ronzio, M.; Semeghini, V.; Andrioletti, V.; Mantovani, R.; Dolfini, D. NF-YA Overexpression in Lung Cancer: LUAD. Genes 2020, 11, 198. [CrossRef]

26. Bezzecchi, E.; Ronzio, M.; Dolfini, D.; Mantovani, R. NF-YA Overexpression in Lung Cancer: LUSC. Genes 2019, 10, 937. [CrossRef] [PubMed]

27. Bezzecchi, E.; Ronzio, M.; Mantovani, R.; Dolfini, D. NF-Y Overexpression in Liver Hepatocellular Carcinoma (HCC). Int. J. Mol. Sci. 2020, 21, 9157. [CrossRef] [PubMed]

28. Puram, S.V.; Tirosh, I.; Parikh, A.S.; Patel, A.P.; Yizhak, K.; Gillespie, S.; Rodman, C.; Luo, C.L.; Mroz, E.A.; Emerick, K.S.; et al. Single-Cell Transcriptomic Analysis of Primary and Metastatic Tumor Ecosystems in Head and Neck Cancer. Cell 2017, 171, 1611-1624.e24. [CrossRef]

29. Love, M.I.; Huber, W.; Anders, S. Moderated estimation of fold change and dispersion for RNA-seq data with DESeq2. Genome Biol. 2014, 15, 550. [CrossRef] [PubMed]

30. Zambelli, F.; Pesole, G.; Pavesi, G. Pscan: Finding over-represented transcription factor binding site motifs in sequences from co-regulated or co-expressed genes. Nucleic Acids Res. 2009, 37, W247-W252. [CrossRef] 
31. Citro, S.; Bellini, A.; Miccolo, C.; Ghiani, L.; Carey, T.E.; Chiocca, S. Synergistic antitumour activity of HDAC inhibitor SAHA and EGFR inhibitor gefitinib in head and neck cancer: A key role for $\Delta$ Np63 $\alpha$. Br. J. Cancer 2019, 120, 658-667. [CrossRef]

32. Brenner, J.C.; Bs, M.P.G.; Kumar, B.; Bs, L.M.S.; Kupfer, R.; Lyons, R.H.; Bradford, C.R.; Carey, T.E. Genotyping of 73 UM-SCC head and neck squamous cell carcinoma cell lines. Head Neck 2009, 32, 417-426. [CrossRef]

33. Ballo, H.; Koldovsky, P.; Hoffmann, T.; Balz, V.; Hildebrandt, B.; Gerharz, C.D.; Bier, H. Establishment and characterization of four cell lines derived from human head and neck squamous cell carcinomas for an autologous tumor-fibroblast in vitro model. Anticancer Res. 1999, 19, 3827-3836.

34. Tang, A.L.; Hauff, S.J.; Owen, J.H.; Bs, M.P.G.; Czerwinski, M.J.; Park, J.J.; Ma, H.W.; Papagerakis, S.; Stoerker, J.; McHugh, J.B.; et al. UM-SCC-104: A New human papillomavirus-16-positive cancer stem cell-containing head and neck squamous cell carcinoma cell line. Head Neck 2012, 34, 1480-1491. [CrossRef] [PubMed]

35. Borcherding, N.; Bormann, N.L.; Voigt, A.P.; Zhang, W. TRGAted: A web tool for survival analysis using protein data in the Cancer Genome Atlas. F1000Research 2018, 7, 1235. [CrossRef]

36. Therneau, T. A Package for Survival Analysis, version 2.38; 2015.

37. Bailey, M.H.; Tokheim, C.; Porta-Pardo, E.; Sengupta, S.; Bertrand, D.; Weerasinghe, A.; Colaprico, A.; Wendl, M.C.; Kim, J.; Reardon, B.; et al. Comprehensive Characterization of Cancer Driver Genes and Mutations. Cell 2018, 173, 371-385.e18. [CrossRef] [PubMed]

38. Repana, D.; Nulsen, J.; Dressler, L.; Bortolomeazzi, M.; Venkata, S.K.; Tourna, A.; Yakovleva, A.; Palmieri, T.; Ciccarelli, F.D. The Network of Cancer Genes (NCG): A comprehensive catalogue of known and candidate cancer genes from cancer sequencing screens. Genome Biol. 2019, 20, 1-12. [CrossRef] [PubMed]

39. Braakhuis, B.J.M.; Snijders, P.J.F.; Keune, W.-J.H.; Meijer, C.J.L.M.; Ruijter-Schippers, H.J.; Leemans, C.R.; Brakenhoff, R.H. Genetic Patterns in Head and Neck Cancers That Contain or Lack Transcriptionally Active Human Papillomavirus. J. Natl. Cancer Inst. 2004, 96, 998-1006. [CrossRef] [PubMed]

40. Chung, C.H.; Gillison, M.L. Human papillomavirus in head and neck cancer: Its role in pathogenesis and clinical implications. Clin. Cancer Res. 2009, 15, 6758-6762. [CrossRef] [PubMed]

41. Rautava, J.; Syrjänen, S. Biology of Human Papillomavirus Infections in Head and Neck Carcinogenesis. Head Neck Pathol. 2012, 6, 3-15. [CrossRef]

42. Jung, A.C.; Briolat, J.; Millon, R.; de Reyniès, A.; Rickman, D.; Thomas, E.; Abecassis, J.; Clavel, C.; Wasylyk, B. Biological and clinical relevance of transcriptionally active human papillomavirus (HPV) infection in oropharynx squamous cell carcinoma. Int. J. Cancer 2010, 126, 1882-1894. [CrossRef] [PubMed]

43. Al Moustafa, A.-E.; Foulkes, W.D.; Benlimame, N.; Wong, A.; Yen, L.; Bergeron, J.; Batist, G.; Alpert, L.; A Alaoui-Jamali, M. E6/E7 proteins of HPV type 16 and ErbB-2 cooperate to induce neoplastic transformation of primary normal oral epithelial cells. Oncogene 2004, 23, 350-358. [CrossRef]

44. Sano, D.; Oridate, N. The molecular mechanism of human papillomavirus-induced carcinogenesis in head and neck squamous cell carcinoma. Int. J. Clin. Oncol. 2016, 21, 819-826. [CrossRef]

45. Gillison, M.L.; Akagi, K.; Xiao, W.; Jiang, B.; Pickard, R.K.; Li, J.; Swanson, B.J.; Agrawal, A.D.; Zucker, M.; Stache-Crain, B.; et al. Human papillomavirus and the landscape of secondary genetic alterations in oral cancers. Genome Res. 2019, 29, 1-17. [CrossRef] [PubMed]

46. Sabatini, M.E.; Chiocca, S. Human papillomavirus as a driver of head and neck cancers. Br. J. Cancer 2019, 122, 306-314. [CrossRef]

47. Citro, S.; Bellini, A.; Medda, A.; Sabatini, M.E.; Tagliabue, M.; Chu, F.; Chiocca, S. Human Papilloma Virus Increases deltaNp63alpha Expression in Head and Neck Squamous Cell Carcinoma. Front. Cell Infect. Microbiol. 2020, 10, 143. [CrossRef]

48. Oldfield, A.J.; Henriques, T.; Kumar, D.; Burkholder, A.B.; Cinghu, S.; Paulet, D.; Bennett, B.D.; Yang, P.; Scruggs, B.S.; Lavender, C.A.; et al. NF-Y controls fidelity of tran-scription initiation at gene promoters through maintenance of the nucleosome-depleted region. Nat. Commun. 2019, 10, 3072. [CrossRef] [PubMed]

49. Cui, H.; Zhang, M.; Wang, Y.; Wang, Y. NF-YC in glioma cell proliferation and tumor growth and its role as an independent predictor of patient survival. Neurosci. Lett. 2016, 631, 40-49. [CrossRef] [PubMed]

50. Jiang, X.; Nevins, J.R.; Shats, I.; Chi, J.-T. E2F1-Mediated Induction of NFYB Attenuates Apoptosis via Joint Regulation of a Pro-Survival Transcriptional Program. PLoS ONE 2015, 10, e0127951. [CrossRef]

51. Frontini, M.; Imbriano, C.; Manni, I.; Mantovani, R. Cell cycle regulation of NF-YC nuclear localization. Cell Cycle 2004, 3, 217-222. [CrossRef]

52. Kahle, J.; Baake, M.; Doenecke, D.; Albig, W. Subunits of the heterotrimeric transcription factor NF-Y are imported into the nucleus by distinct pathways involving importin beta and importin 13. Mol. Cell Biol. 2005, 25, 5339-5354. [CrossRef]

53. Liu, Z.; Tao, H. Small nucleolar RNA host gene 3 facilitates cell proliferation and migration in oral squamous cell carcinoma via targeting nuclear transcription factor Y subunit gamma. J. Cell Biochem. 2020, 121, 2150-2158. [CrossRef] [PubMed]

54. Balz, V.; Scheckenbach, K.; Götte, K.; Bockmühl, U.; Petersen, I.; Bier, H. Is the p53 inactivation frequency in squamous cell carcinomas of the head and neck underestimated? Analysis of p53 exons 2-11 and human papillomavirus 16/18 E6 transcripts in 123 unselected tumor specimens. Cancer Res. 2003, 63, 1188-1191. [PubMed]

55. Wilkie, M.; Lau, A.; Vlatković, N.; Jones, T.; Boyd, M. Tumour metabolism in squamous cell carcinoma of the head and neck: An in-vitro study of the consequences of TP53 mutation and therapeutic implications. Lancet 2015, 385, S101. [CrossRef] 
56. Zhou, G.; Liu, Z.; Myers, J.N. TP53Mutations in Head and Neck Squamous Cell Carcinoma and Their Impact on Disease Progression and Treatment Response. J. Cell. Biochem. 2016, 117, 2682-2692. [CrossRef]

57. Cancer Genome Atlas Network. Comprehensive genomic characterization of squamous cell lung Cancers. Nature 2012, 489, 519-525.

58. Di Agostino, S.; Strano, S.; Emiliozzi, V.; Zerbini, V.; Mottolese, M.; Sacchi, A.; Blandino, G.; Piaggio, G. Gain of function of mutant p53: The mutant $\mathrm{p} 53$ /NF-Y protein complex reveals an aberrant transcriptional mechanism of cell cycle regulation. Cancer Cell 2006, 10, 191-202. [CrossRef] [PubMed]

59. Liu, K.; Ling, S.; Lin, W.-C. TopBP1 Mediates Mutant p53 Gain of Function through NF-Y and p63/p73. Mol. Cell Biol. 2011, 31, 4464-4481. [CrossRef]

60. Huang, X.; Zhang, Y.; Tang, Y.; Butler, N.; Kim, J.; Guessous, F.; Schiff, D.; Mandell, J.; Abounader, R. A novel PTEN/mutant p53/c-Myc/Bcl-XL axis mediates con-text-dependent oncogenic effects of PTEN with implications for cancer prognosis and therapy. Neoplasia 2013, 15, 952-965. [CrossRef]

61. Weissmueller, S.; Manchado, E.; Saborowski, M.; Morris, J.P.; Wagenblast, E.; Davis, C.A.; Moon, S.-H.; Pfister, N.T.; Tschaharganeh, D.F.; Kitzing, T.; et al. Mutant p53 Drives Pancreatic Cancer Metastasis through Cell-Autonomous PDGF Receptor $\beta$ Signaling. Cell 2014, 157, 382-394. [CrossRef]

62. Alam, S.K.; Yadav, V.K.; Bajaj, S.; Datta, A.; Dutta, S.K.; Bhattacharyya, M.; Bhattacharya, S.; Debnath, S.; Roy, S.; Boardman, L.A.; et al. DNA damage-induced ephrin-B2 reverse signaling promotes chemoresistance and drives EMT in colorectal carcinoma harboring mutant p53. Cell Death Differ. 2015, 23, 707-722. [CrossRef]

63. Chen, X.; Ma, H.; Wang, Z.; Zhang, S.; Yang, H.; Fang, Z. EZH2 Palmitoylation Mediated by ZDHHC5 in p53-Mutant Glioma Drives Malignant Development and Progression. Cancer Res. 2017, 77, 4998-5010. [CrossRef]

64. Benatti, P.; Basile, V.; Dolfini, D.; Belluti, S.; Tomei, M.; Imbriano, C. NF-Y loss triggers p53 stabilization and apoptosis in HPV18-positive cells by affecting E6 transcription. Oncotarget 2016, 7, 45901-45915. [CrossRef] [PubMed]

65. Li, C.; Johnson, D.E. Liberation of functional p53 by proteasome inhibition in human papilloma virus-positive head and neck squamous cell carcinoma cells promotes apoptosis and cell cycle arrest. Cell Cycle 2013, 12, 923-934. [CrossRef] [PubMed]

66. Bullenkamp, J.; Raulf, N.; Ayaz, B.; Walczak, H.; Kulms, D.; Odell, E.; Thavaraj, S.; Tavassoli, M. Bortezomib sensitises TRAILresistant HPV-positive head and neck cancer cells to TRAIL through a caspase-dependent, E6-independent mechanism. Cell Death Dis. 2014, 5, e1489. [CrossRef] [PubMed]

67. Yuan, C.-H.; Filippova, M.; Krstenansky, J.L.; Duerksen-Hughes, P.J. Flavonol and imidazole derivatives block HPV16 E6 activities and reactivate apoptotic pathways in HPV+ cells. Cell Death Dis. 2016, 7, e2060. [CrossRef] [PubMed]

68. Gatti, V.; Fierro, C.; Annicchiarico-Petruzzelli, M.; Melino, G.; Peschiaroli, A. $\Delta$ Np63 in squamous cell carcinoma: Defining the oncogenic routes affecting epigenetic landscape and tumour microenvironment. Mol. Oncol. 2019, 13, 981-1001. [CrossRef]

69. Romano, R.A.; Birkaya, B.; Sinha, S. Defining the regulatory elements in the proximal promoter of DeltaNp63 in keratinocytes: Potential roles for Sp1/Sp3, NF-Y, and p63. J. Investig. Dermatol. 2006, 126, 1469-1479. [CrossRef]

70. Dolfini, D.; Minuzzo, M.; Pavesi, G.; Mantovani, R. The Short Isoform of NF-YA Belongs to the Embryonic Stem Cell Transcription Factor Circuitry. Stem Cells 2012, 30, 2450-2459. [CrossRef]

71. Fougner, C.; Bergholtz, H.; Norum, J.H.; Sørlie, T. Re-definition of claudin-low as a breast cancer phenotype. Nat. Commun. 2020, 11, 1-11. [CrossRef] [PubMed]

72. Sahai, E.; Astsaturov, I.; Cukierman, E.; DeNardo, D.G.; Egeblad, M.; Evans, R.M.; Fearon, D.; Greten, F.R.; Hingorani, S.R.; Hunter, T.; et al. A framework for advancing our understanding of cancer-associated fibroblasts. Nat. Rev. Cancer 2020, 20, 174-186. [CrossRef]

73. Jeganathan, S.; Wendt, M.; Kiehstaller, S.; Brancaccio, D.; Kuepper, A.; Pospiech, N.; Carotenuto, A.; Novellino, E.; Hennig, S.; Grossmann, T.N. Constrained Peptides with Fine-Tuned Flexibility Inhibit NF-Y Transcription Factor Assembly. Angew. Chem. Int. Ed. 2019, 58, 17351-17358. [CrossRef] [PubMed]

74. Nardone, V.; Chaves-Sanjuan, A.; Lapi, M.; Airoldi, C.; Saponaro, A.; Pasqualato, S.; Dolfini, D.; Camilloni, C.; Bernardini, A.; Gnesutta, N.; et al. Structural Basis of Inhibition of the Pioneer Transcription Factor NF-Y by Suramin. Cells 2020, 9, 2370. [CrossRef] [PubMed] 